\title{
POWER ESTIMATES FOR RUIN PROBABILITIES
}

\author{
HARRI NYRHINEN, ${ }^{*}$ University of Helsinki
}

\begin{abstract}
Let $X_{1}, X_{2}, \ldots$ be real-valued random variables. For $u>0$, define the time of ruin $T=T(u)$ by $T=\inf \left\{n: X_{1}+\cdots+X_{n}>u\right\}$ or $T=\infty$ if $X_{1}+\cdots+X_{n} \leq u$ for every $n=1,2, \ldots$ We are interested in the ruin probabilities of general processes $\left\{X_{n}\right\}$ for large $u$. In the presence of heavy tails, one often finds power estimates. Our objective is to specify the associated powers and provide the crude estimate $\mathrm{P}(T \leq x u) \approx u^{-R(x)}$ for large $u$, for a given $x \in \mathbb{R}$. The rate $R(x)$ will be described by means of tails of partial sums and maxima of $\left\{X_{n}\right\}$. We also extend our results to the case of the infinite time horizon.
\end{abstract}

Keywords: Ruin probability; heavy tail; conditional independence; large deviation

2000 Mathematics Subject Classification: Primary 60G40

Secondary 60F10

\section{Introduction}

Let $X_{1}, X_{2}, \ldots$ be a sequence of random variables and $u$ a positive real number. We interpret $X_{n}$ as the discounted net payoff of the year $n$ and $u$ as the initial capital of an insurance company. Write $S_{n}=X_{1}+\cdots+X_{n}$ for $n=1,2, \ldots$ The time of ruin $T=T(u)$ is, by definition,

$$
T=\left\{\begin{array}{l}
\inf \left\{n: S_{n}>u\right\} \\
\infty \quad \text { if } S_{n} \leq u \text { for every } n=1,2, \ldots
\end{array}\right.
$$

We are interested in the ruin probabilities for large $u$. The main interest is in finite time horizons but the probability $\mathrm{P}(T<\infty)$ will also be studied.

General processes $\left\{X_{n}\right\}$ are of interest in insurance mathematics. In basic models, $X_{n}$ describes the difference between the claims and the premiums in the year $n$. It is natural to allow dependence between the consecutive years because the claim history often affects the future premiums. Also, the claim process itself can have dependent increments, for example because of economic cycles. By discounting the net payoffs, we can consider models in which investments in risky assets are allowed. This feature further complicates the structure of the process. We refer the reader to Daykin et al. (1994) for more information about practical models in this context.

In general models, it can be difficult to find sharp estimates for the ruin probabilities even in the asymptotic sense. However, to obtain an idea about the magnitudes, we can consider

Received 19 January 2005; revision received 7 April 2005.

* Postal address: Department of Mathematics and Statistics, University of Helsinki, PO Box 68, Helsinki, FIN-00014, Finland. Email address: harri.nyrhinen@helsinki.fi 
rate results. For example, for a given $x>0$, it is often possible to justify the crude estimate $\mathrm{P}(T \leq x u) \approx \mathrm{e}^{-r(x) u}$ and specify the decay rate $r(x)$. The precise meaning of this is that

$$
\lim _{u \rightarrow \infty} u^{-1} \log \mathrm{P}(T \leq x u)=-r(x) .
$$

See Collamore (1998) and Nyrhinen (1998) for results of this type.

In models in which increments are heavy tailed, limit (1.1) is not very useful. It is more instructive to consider alternative scalings. Suppose that there exists the limit

$$
\lim _{u \rightarrow \infty}(\log u)^{-1} \log \mathrm{P}(T \leq x u)=-R(x),
$$

with $R(x) \in(0, \infty)$. This limit justifies the power estimate $\mathrm{P}(T \leq x u) \approx u^{-R(x)}$ for large $u$. Additionally, the form of the rate may reveal which parts of the model are critical to the risk of ruin. We will study examples in which heavy-tailed claims, cyclic behaviour, and risky investments feature. By considering rates such as (1.2), we obtain various extensions of earlier results.

For heavy-tailed processes, connections between the time of ruin, partial sums, and maxima have been stated in many studies. We refer the reader to Embrechts et al. (1997) for general background. Results similar to (1.2) can be found in Duffy et al. (2003). They considered infinite-time ruin probabilities with general scalings. The results of their paper apply to a class of heavy-tailed processes but do not cover power tails.

Power estimates for finite-time ruin probabilities are included in many papers, in particular in the case of processes with regularly varying tails. Asmussen and Klüppelberg (1996) derived sharp approximations for random walks. Extensions were given in Asmussen (1998), where varying premiums and riskless investments were allowed. Nyrhinen (2001) derived rate results for processes including a submodel for risky investments. Refinements were obtained in Tang and Tsitsiashvili (2003), where, among other results, processes were classified in terms of insurance and financial risks. Finally, Hu (2004) obtained power estimates for quick ruin in a generalized classical model.

Precise power estimates for infinite-time ruin probabilities were found in Embrechts and Veraverbeke (1982), who considered standard classical models. Similar results in a Markovian environment were given in Asmussen et al. (1994). Further extensions were obtained in Asmussen et al. (1999), where general dependence structures were allowed. Mikosch and Samorodnitsky (2000) derived sharp approximations in a model where the increments are moving averages of independent, identically distributed random variables. Precise estimates are also known for appropriately perturbed classical models; we refer the reader to Cai and Tang (2004) and the references therein. A submodel for the returns on the investments is included in several papers: Asmussen (1998) and Klüppelberg and Stadtmüller (1998) obtained sharp estimates in the case where the rate of return is deterministic, and Paulsen (2002) extended these results by allowing for riskiness of the returns. More general rate results, in a similar environment, were derived in Nyrhinen (1999).

The paper is organized as follows. Main results are stated in Section 2. Examples are considered in Section 3. In Section 4, we give the proofs of the main results and, in Section 5, we give the technical background for the examples.

\section{Main results}

Let $X_{1}, X_{2}, \ldots$ be real-valued random variables on a fixed probability space and write $S_{n}=X_{1}+\cdots+X_{n}$ and $M_{n}=\max \left(X_{1}, \ldots, X_{n}\right)$ for $n=1,2, \ldots$ Let $u$ be the initial capital 
and $T$ the time of ruin, as described in Section 1. Our objective is to derive estimates for ruin probabilities in terms of tail probabilities of the processes $\left\{S_{n}\right\}$ and $\left\{M_{n}\right\}$. Part of this task can be carried out without an explicit specification of the dependence structure - this is the content of Section 2.1. Refinements are given in Section 2.2, where mixtures of random vectors with independent components are studied.

\subsection{General bounds for ruin probabilities}

In this section, we consider finite-time ruin probabilities for general processes. Our basic assumption is that the left-hand tails of $\left\{X_{n}\right\}$ are not too heavy. Two-sided bounds will be derived in terms of partial sums; we obtain lower bounds in terms of maxima.

In order to describe ruin probabilities, we introduce decay rates associated with the processes $\left\{S_{n}\right\}$ and $\left\{M_{n}\right\}$. For $y>0$, define the parameters $\bar{R}_{S}(y)$ and $\underline{R}_{S}(y)$ according to

$$
\begin{gathered}
\lim _{\delta \rightarrow 0+} \limsup _{n \rightarrow \infty}(\log n)^{-1} \log \mathrm{P}\left(S_{n}>n(y-\delta)\right)=-\bar{R}_{S}(y), \\
\lim _{\delta \rightarrow 0+} \liminf _{n \rightarrow \infty}(\log n)^{-1} \log \mathrm{P}\left(S_{n}>n(y+\delta)\right)=-\underline{R}_{S}(y) .
\end{gathered}
$$

If both parameters are finite then, for every given $\varepsilon>0$,

$$
n^{-\underline{R}_{S}(y)-\varepsilon} \leq \mathrm{P}\left(S_{n}>n y\right) \leq n^{-\bar{R}_{S}(y)+\varepsilon}
$$

for large $n$. Clearly, $\bar{R}_{S}$ and $\underline{R}_{S}$ are increasing in $y$ and $\bar{R}_{S}(y) \leq \underline{R}_{S}(y)$ for every $y>0$. It is also easy to see that $\bar{R}_{S}$ is continuous from the left and that $\underline{R}_{S}$ is continuous from the right. For the maxima, we define the global parameters $\bar{R}_{M}$ and $\underline{R}_{M}$ according to

$$
\begin{aligned}
& \lim _{\delta \rightarrow 0+} \limsup _{n \rightarrow \infty}(\log n)^{-1} \log \mathrm{P}\left(M_{n}>n^{1-\delta}\right)=-\bar{R}_{M}, \\
& \lim _{\delta \rightarrow 0+} \liminf _{n \rightarrow \infty}(\log n)^{-1} \log \mathrm{P}\left(M_{n}>n^{1+\delta}\right)=-\underline{R}_{M} .
\end{aligned}
$$

We begin with a simple lemma concerning the lower bounds.

Lemma 2.1. Assume that

$$
\lim _{\delta \rightarrow 0+} \limsup _{n \rightarrow \infty}(\log n)^{-1} \log \mathrm{P}\left(\sum_{i=1}^{n}\left|X_{i}\right| \mathbf{1}\left(X_{i} \leq 0\right)>n^{1+\delta}\right)<-\underline{R}_{M} .
$$

Then, for every $x>0$, we have

$$
\liminf _{u \rightarrow \infty}(\log u)^{-1} \log \mathrm{P}(T \leq x u) \geq-\underline{R}_{S}(1 / x) \geq-\underline{R}_{M} .
$$

The first inequality of (2.4) actually holds without any conditions.

We next give sufficient conditions for (2.4) that are stronger than (2.3) but more elementary. A further advance is that, in essence, the first inequality can be reversed.

Theorem 2.1. Assume that

$$
\sup _{n \in \mathbb{N}} \mathrm{E}\left(\left|X_{n}\right|^{t} \mathbf{1}\left(X_{n} \leq 0\right)\right)<\infty
$$

for every $t>0$, and let $x>0$. Then (2.4) holds and

$$
\limsup _{u \rightarrow \infty}(\log u)^{-1} \log \mathrm{P}(T \leq x u) \leq-\bar{R}_{S}(1 / x) .
$$




\subsection{Refinements of the upper bounds}

The main objective of this section is to derive upper bounds for ruin probabilities in terms of maxima. We also extend our results to the case of the infinite time horizon.

We will study models in which the joint distributions of the increments are mixtures of distributions of random vectors with independent components. Specifically, let $\left(\eta_{1}, \eta_{2}, \ldots\right)$ be a real-valued stochastic process and let $H_{n}$ be its joint distribution function. We associate a random variable $\xi_{v}$ and its distribution function $G_{v}$ with each $v \in \mathbb{R}$, and assume that, for every fixed $x \in \mathbb{R}$, the map $v \mapsto G_{v}(x)$ is measurable. We consider the model in which, for every $x_{1}, \ldots, x_{n} \in \mathbb{R}$ and $y_{1}, \ldots, y_{n} \in \mathbb{R}$, we have

$$
\begin{aligned}
\mathrm{P}\left(X_{1}\right. & \left.\leq x_{1}, \ldots, X_{n} \leq x_{n}, \eta_{1} \leq y_{1}, \ldots, \eta_{n} \leq y_{n}\right) \\
& =\int_{v_{1} \leq y_{1}, \ldots, v_{n} \leq y_{n}} G_{v_{1}}\left(x_{1}\right) \cdots G_{v_{n}}\left(x_{n}\right) \mathrm{d} H_{n}\left(v_{1}, \ldots, v_{n}\right) .
\end{aligned}
$$

Thus, conditionally on $\eta_{1}=v_{1}, \ldots, \eta_{n}=v_{n}$, the variables $X_{1}, \ldots, X_{n}$ are independent and the distribution function of $X_{i}$ is $G_{v_{i}}$, for every $i \leq n$.

We assume that the mean

$$
\mu(v)=\mathrm{E}\left(\xi_{v}\right)=\int_{x \in \mathbb{R}} x \mathrm{~d} G_{v}(x)
$$

exists for every $v \in \mathbb{R}$ and that $\mathrm{E}\left(X_{n}\right)$ exists for every $n \in \mathbb{N}$. Let $f_{i}: \mathbb{R} \rightarrow \mathbb{R}$ be a measurable map for $i=1,2, \ldots$ If

$$
\mathrm{E}\left(f_{1}\left(X_{1}\right) \cdots f_{n}\left(X_{n}\right)\right)
$$

exists then

$$
\mathrm{E}\left(f_{1}\left(X_{1}\right) \cdots f_{n}\left(X_{n}\right) \mid \eta_{1}, \ldots, \eta_{n}\right)=\prod_{i=1}^{n} \int_{x_{i} \in \mathbb{R}} f_{i}\left(x_{i}\right) \mathrm{d} G_{\eta_{i}}\left(x_{i}\right)
$$

almost surely. In particular, $\mu\left(\eta_{i}\right)$ is a version of $\mathrm{E}\left(X_{i} \mid \eta_{1}, \ldots, \eta_{n}\right)$, for $i \leq n$. For $a \in \mathbb{R}$, we define the parameter $p(a)$ according to

$$
\limsup _{n \rightarrow \infty}(\log n)^{-1} \log \mathrm{P}\left(\mu\left(\eta_{1}\right)+\cdots+\mu\left(\eta_{n}\right)>n a\right)=-p(a) .
$$

Theorem 2.2. Assume that

$$
\sup _{v \in \mathbb{R}} \mathrm{E}\left(\left|\xi_{v}\right|^{t} \mathbf{1}\left(\xi_{v} \leq 0\right)\right)<\infty
$$

for every $t>0$, and let $a>0$. Then $\bar{R}_{S}(y) \geq \min \left(p(a), \bar{R}_{M}\right)$ for every $y>a$ and

$$
\limsup _{u \rightarrow \infty}(\log u)^{-1} \log \mathrm{P}(T \leq x u) \leq-\min \left(p(a), \bar{R}_{M}\right)
$$

for every $x<1 / a$.

Now consider infinite-time ruin probabilities. Obviously, (2.8) implies (2.5). Thus, under (2.8), we have (2.4) and, hence, the following lower bound:

$$
\liminf _{u \rightarrow \infty}(\log u)^{-1} \log \mathrm{P}(T<\infty) \geq-\underline{R}_{M} .
$$


Theorem 2.3. Assume that (2.8) is satisfied for every $t>0$ and that

$$
\lim _{a \rightarrow 0-} p(a) \geq \bar{R}_{M}+1 .
$$

Then

$$
\limsup _{u \rightarrow \infty}(\log u)^{-1} \log \mathrm{P}(T<\infty) \leq-\bar{R}_{M} .
$$

We end the section by giving the starting point of a method of finding the parameters $\bar{R}_{M}$ and $\underline{R}_{M}$. Observe that, for every $n \in \mathbb{N}$ and $y>0$, we always have the simple bound

$$
\mathrm{P}\left(M_{n}>n^{y}\right) \leq \sum_{i=1}^{n} \mathrm{P}\left(X_{i}>n^{y}\right) .
$$

Theorem 2.4. Assume that

$$
\sup _{v \in \mathbb{R}} \mathrm{E}\left(\xi_{v}^{t} \mathbf{1}\left(\xi_{v} \geq 0\right)\right)<\infty
$$

for some $t>1$. Then, for y sufficiently close to 1 ,

$$
\mathrm{P}\left(M_{n}>n^{y}\right) \sim \sum_{i=1}^{n} \mathrm{P}\left(X_{i}>n^{y}\right)
$$

when $n$ tends to infinity.

Remark 2.1. The mixing sequence $\left\{\eta_{n}\right\}$ could take values in $\mathbb{R}^{d}$, for arbitrary $d \in \mathbb{N}$, with obvious changes in conditions (2.8) and (2.14). A simple useful two-dimensional extension is obtained by taking $\eta_{n}=\left(\eta_{n}^{\prime}, n\right)$, where $\left\{\eta_{n}^{\prime}\right\}$ is real valued. Then the $\xi$-variables can be inhomogeneous in time. We have chosen to work with $\mathbb{R}$ for simplicity and because it seems to be a reasonable choice for many interesting processes.

\section{Examples}

Here, we apply the results of Section 2 to four classes of process. We only describe the models and the estimates for the ruin probabilities. The technical background is given in Section 5 .

In the first two examples, we study basic models such as random walks and simple Markovian structures. Under suitable conditions, estimates for ruin probabilities can be given in terms of the maxima. This hints that ruin typically occurs because of a single large increment. In comparison with many related papers, we need fewer regularity conditions on the right-hand tails of the distributions. The most common assumption in earlier studies is that of regular variation.

In the third example, we consider a model in which the increments are heavy tailed and good and bad business periods occur randomly. The lengths of the periods are also assumed to be heavy tailed. This assumption has a theoretical nature. On a short time horizon, a single large increment can be seen as the source of ruin, whereas, on a longer time horizon, ruin typically occurs because of a long bad period.

The fourth example deals with a model in which investments in risky assets are allowed. The net payoffs are assumed to be heavy tailed. In this example, both the risky investments and the heavy tails of the net payoffs have an essential influence on ruin probabilities on a short time horizon. On a longer time horizon, the risk associated with the investments dominates. 
For any distribution function $F$, write $\bar{F}=1-F$. Furthermore, define the parameters $\bar{\alpha}_{F}$ and $\underline{\alpha}_{F}$ according to

$$
\limsup _{x \rightarrow \infty}(\log x)^{-1} \log \bar{F}(x)=-\bar{\alpha}_{F}
$$

and

$$
\liminf _{x \rightarrow \infty}(\log x)^{-1} \log \bar{F}(x)=-\underline{\alpha}_{F} .
$$

Example 3.1. Assume that $X_{1}, X_{2}, \ldots$ are independent random variables. Suppose that the mean $m_{n}=\mathrm{E}\left(X_{n}\right)$ exists for every $n$ and that (2.5) is satisfied for every $t>0$. Let

$$
m=\limsup _{n \rightarrow \infty} n^{-1}\left(m_{1}+\cdots+m_{n}\right) .
$$

In insurance models, it is usually assumed that $m$ is negative. The interpretation is that the accumulated premiums include a positive safety loading. Then, for every $x>0$, we have (2.4) and

$$
\limsup _{u \rightarrow \infty}(\log u)^{-1} \log \mathrm{P}(T \leq x u) \leq-\bar{R}_{M} .
$$

Inequalities (2.10) and (2.12) also hold. If $m$ is positive then (2.4) and (3.1) hold for every $x<1 / m$.

Assume, in particular, that $X_{1}, X_{2}, \ldots$ are identically distributed, with common distribution function $F$. Suppose that $\mathrm{E}\left(X_{1}\right)$ is negative and that $\bar{\alpha}_{F}>1$. Then $\bar{R}_{M}=\bar{\alpha}_{F}-1$ and $\underline{R}_{M}=\underline{\alpha}_{F}-1$. Instead of (2.5), assume that

$$
\mathrm{E}\left(\left|X_{1}\right|^{t} \mathbf{1}\left(X_{1} \leq 0\right)\right)<\infty
$$

for some $t>\underline{\alpha}_{F}$. Let $x>0$ be arbitrary. Then (2.4), (3.1), (2.10), and (2.12) hold.

Furthermore, assume that $\bar{F}$ is regularly varying with index $\alpha>1$. Then $\bar{R}_{M}=\underline{R}_{M}=\alpha-1$. Hence, (1.2) holds with $R(x)=\alpha-1$. This case is well understood from earlier studies; see, for example, Asmussen and Klüppelberg (1996). The rate $R(x)$ does not depend on $x$. To see differences between the time horizons, we can consider the probability $\mathrm{P}\left(T \leq u^{x}\right)$ for a given $x \in(0,1)$. We then have

$$
\lim _{u \rightarrow \infty}(\log u)^{-1} \log \mathrm{P}\left(T \leq u^{x}\right)=x-\alpha .
$$

Limits of this type were derived in $\mathrm{Hu}$ (2004). We give an alternative proof in Section 5.

Example 3.2. Consider the model described in Section 2.2. Assume that $\left\{\eta_{n}\right\}$ is an aperiodic, irreducible Markov chain on a finite state space $E=\{1, \ldots, d\}$. Then $\left\{\left(\eta_{n}, S_{n}\right)\right\}$ is a Markov additive process. Sharp estimates for infinite-time ruin probabilities in a similar set-up can be found in Asmussen et al. (1994).

We assume that (2.8) is satisfied for every $t>0$ and, furthermore, that $\bar{\alpha}_{G_{j}}=\underline{\alpha}_{G_{j}}$ and $\bar{\alpha}_{G_{j}}>1$ for every $j \in E$. Let $\{\pi(j), j \in E\}$ be the invariant measure of $\left\{\eta_{n}\right\}$ and let

$$
m=\sum_{j \in E} \pi(j) \mu(j) .
$$

We assume that $m$ is negative. Then (1.2) holds for every $x>0$, with

$$
R(x)=\bar{R}_{M}=\underline{R}_{M}=\min \left\{\bar{\alpha}_{G_{j}}, j \in E\right\}-1 .
$$

Inequalities (2.10) and (2.12) also hold. As in Example 3.1, we can prove (3.3) with $\alpha$ replaced by $\bar{R}_{M}+1$. This extends the result of $\mathrm{Hu}(2004)$. 
Example 3.3. Again consider the model of Section 2.2. We assume that the $\eta$-variables can take only the values 1 and 2 . The value 1 describes a good year and the value 2 a bad year. Suppose that $\bar{\alpha}_{G_{1}}=\underline{\alpha}_{G_{1}}=\bar{\alpha}_{G_{2}}=\underline{\alpha}_{G_{2}}=: \alpha$. We assume that $\alpha \in(1, \infty)$ and that $(2.8)$ is satisfied for every $t>0$. Suppose that $\mu(1)<0$ and $\mu(2)>0$. Thus, in the mean, the net payoff is negative in the good years and positive in the bad years. We also assume that $\mu(1)+\mu(2)<0$.

To complete the model description, we must specify the structure of the sequence $\left\{\eta_{n}\right\}$. Let $v_{1}, v_{2}, \ldots$ be independent and identically distributed random variables and let $F$ be the distribution function of $\nu_{1}$. Suppose that $\bar{\alpha}_{F}=\underline{\alpha}_{F}=: \beta$. We assume that $\beta \in(1, \alpha)$ and that $\sum_{n=1}^{\infty} \mathrm{P}\left(v_{1}=n\right)=1$. Consider the model in which

$$
\begin{aligned}
\eta_{1}, \ldots, \eta_{v_{1}} & =2, \\
\eta_{v_{1}+1}, \ldots, \eta_{v_{1}+v_{2}} & =1, \\
\eta_{v_{1}+v_{2}+1}, \ldots, \eta_{v_{1}+v_{2}+v_{3}} & =2, \\
\eta_{v_{1}+v_{2}+v_{3}+1}, \ldots, \eta_{v_{1}+v_{2}+v_{3}+v_{4}} & =1,
\end{aligned}
$$

This structure produces simple stochastic cyclic behaviour: there are bad years for a random time $v_{1}$, good years for a random time $v_{2}$, and so on. The long-run mean of the accumulated net payoff is negative, since we assumed that $\mu(1)+\mu(2)<0$.

In the model described above, (1.2) holds with $R(x)=\alpha-1$ for every $x<1 / \mu(2)$. This corresponds to the rate associated with maxima. Ruin can be seen to occur because of a single large increment. For $x>1 / \mu(2),(1.2)$ holds with $R(x)=\beta-1$. The proof hints that, in this case, ruin typically occurs because of the number of bad years considerably exceeds its typical value. The lengths of the bad periods are heavy tailed, which indicates an occurrence of a single long bad period.

Example 3.4. Assume that the increments take the form

$$
X_{n}=A_{1} \cdots A_{n-1} B_{n}
$$

for $n \in \mathbb{N}$, where both $\left\{A_{n}\right\}$ and $\left\{B_{n}\right\}$ are sequences of independent, identically distributed random variables. Suppose that these sequences are independent and that $\mathrm{P}\left(A_{1}>0\right)=1$. The $A$-variables describe discount factors associated with the returns on the investments, while the $B$-variables describe net payoffs. See Nyrhinen (1999) for more details concerning their interpretations.

Let $F$ be the distribution function of $B_{1}$. Assume that $\bar{\alpha}_{F}=\underline{\alpha}_{F}=: \alpha$ and that $\alpha \in(1, \infty)$. Suppose that

$$
\mathrm{E}\left(\left|B_{1}\right|^{t} \mathbf{1}\left(B_{1} \leq 0\right)\right)<\infty
$$

for some $t>\alpha$. Let $c(t)=\log \mathrm{E}\left(A_{1}^{t}\right)$ for $t \in \mathbb{R}$ and let

$$
c^{*}(v)=\sup \{t v-c(t), t \in \mathbb{R}\}
$$

for $v \in \mathbb{R}$. Hence, $c$ is the cumulant-generating function of $\log A_{1}$ and $c^{*}$ is the FenchelLegendre transform of $c$. We assume that $c(t)<\infty$ for some $t>\alpha$ and that there exists a $w \in(0, \alpha)$ such that $c(w)=0$. For simplicity, we assume that, for every $z>0$, there exists a $t>0$ such that $c^{\prime}(t)=z$. 
Let $x>0$ and write

$$
\rho(x)= \begin{cases}\alpha-x c(\alpha) & \text { if } x \leq \frac{1}{c^{\prime}(\alpha)}, \\ x c^{*}\left(\frac{1}{x}\right) & \text { if } x \in\left(\frac{1}{c^{\prime}(\alpha)}, \frac{1}{c^{\prime}(w)}\right), \\ w & \text { if } x \geq \frac{1}{c^{\prime}(w)} .\end{cases}
$$

Then

$$
\lim _{u \rightarrow \infty}(\log u)^{-1} \log \mathrm{P}(T \leq x \log u)=-\rho(x) .
$$

Observe that the time horizon in (3.5) is $x \log u$ instead of $x u$. For $x>1 / c^{\prime}(\alpha)$, this result is known from Nyrhinen (2001) and the rate $\rho(x)$ depends only on the discount factors. For $x<1 / c^{\prime}(\alpha)$, it also depends on the tails of the net payoffs. By letting $x$ tend to 0 , we see that these tails dominate on a very short time horizon. This complements results of Tang and Tsitsiashvili (2003, Section 5), where fixed time periods were considered. Tang and Tsitsiashvili (2004) obtained results similar to (3.5) in the case that $w>\alpha$, in which the limit (3.5) typically depends only on the net payoffs (see Tang and Tsitsiashvili (2004, Corollary 3.1)).

\section{Proofs of the main results}

We will use the following limit result repeatedly. Let $f_{1}, \ldots, f_{m}$ be real-valued, nonnegative functions on $(0, \infty)$, and let

$$
a_{j}=\limsup _{x \rightarrow \infty}(\log x)^{-1} \log f_{j}(x)
$$

for $j=1, \ldots, m$. Then

$$
\limsup _{x \rightarrow \infty}(\log x)^{-1} \log \left(f_{1}(x)+\cdots+f_{m}(x)\right)=\max \left(a_{1}, \ldots, a_{m}\right) .
$$

For the proof, we refer the reader to Dembo and Zeitouni (1998, Lemma 1.2.15).

Proof of Lemma 2.1. Consider the first inequality of (2.4). Let $u$ be large and let $y \in(0, x)$. Then

$$
\mathrm{P}(T \leq x u) \geq \mathrm{P}\left(S_{\lfloor y u\rfloor}>u\right)
$$

(we denote by $\lfloor c\rfloor$ the integer part of $c \geq 0$ ). Hence, for a given $\varepsilon \in(0, y)$, we have

$$
(\log u)^{-1} \log \mathrm{P}(T \leq x u) \geq \frac{\log \lfloor y u\rfloor}{\log u}(\log \lfloor y u\rfloor)^{-1} \log \mathrm{P}\left(S_{\lfloor y u\rfloor}>\frac{\lfloor y u\rfloor}{y-\varepsilon}\right) .
$$

Thus,

$$
\liminf _{u \rightarrow \infty}(\log u)^{-1} \log \mathrm{P}(T \leq x u) \geq \liminf _{n \rightarrow \infty}(\log n)^{-1} \mathrm{P}\left(S_{n}>\frac{n}{y-\varepsilon}\right) .
$$

We obtain the first inequality of (2.4) from the fact that $y \in(0, x)$ and $\varepsilon \in(0, y)$ are arbitrary.

Consider the second inequality of (2.4). We must show that $\underline{R}_{S}(y) \leq \underline{R}_{M}$ for every $y>0$. We can assume that $\underline{R}_{M}$ is finite. For a given $\delta>0$, we have

$$
\begin{aligned}
\mathrm{P}\left(S_{n}>n y\right) & \geq \mathrm{P}\left(M_{n}>n^{1+\delta}, S_{n}-M_{n}>-n^{1+\delta / 2}\right) \\
& \geq \mathrm{P}\left(M_{n}>n^{1+\delta}\right)-\mathrm{P}\left(S_{n}-M_{n} \leq-n^{1+\delta / 2}\right)
\end{aligned}
$$


for large $n$. Since

$$
\left\{S_{n}-M_{n} \leq-n^{1+\delta / 2}\right\} \subseteq\left\{\sum_{i \leq n}\left|X_{i}\right| \mathbf{1}\left(X_{i} \leq 0\right) \geq n^{1+\delta / 2}\right\},
$$

the second inequality of (2.4) follows from (2.3) and (4.2).

Proof of Theorem 2.1. By Lemma 2.1, the first inequality of (2.4) holds without conditions. Consider the second inequality. We can assume that $\underline{R}_{M}$ is finite and then show that (2.3) holds. Let $t>0$ and $\delta>0$. By Chebyshev's inequality, we have

$$
\mathrm{P}\left(\left|X_{i}\right| \mathbf{1}\left(X_{i} \leq 0\right)>n^{\delta}\right) \leq C(t) n^{-\delta t}
$$

for every $i \in \mathbb{N}$, where $C(t)$ is the supremum in (2.5). Hence,

$$
\mathrm{P}\left(\sum_{i \leq n}\left|X_{i}\right| \mathbf{1}\left(X_{i} \leq 0\right)>n^{1+\delta}\right) \leq C(t) n^{1-\delta t} .
$$

By letting $t$ tend to infinity, we see that

$$
\limsup _{n \rightarrow \infty}(\log n)^{-1} \log \mathrm{P}\left(\sum_{i \leq n}\left|X_{i}\right| \mathbf{1}\left(X_{i} \leq 0\right)>n^{1+\delta}\right)=-\infty .
$$

Thus, (2.3) holds. The second inequality of (2.4) follows from Lemma 2.1.

Consider (2.6). We can assume that $\bar{R}_{S}(1 / x)>0$. In the following proof, we also assume that $\bar{R}_{S}(1 / x)<\infty$. A similar approach leads to (2.6) in the case in which $\bar{R}_{S}(1 / x)=\infty$. Let $\varepsilon>0$ be small and let $\varepsilon^{\prime} \in(0, \varepsilon)$. Furthermore, let $j \in\left[0, u^{\varepsilon}-1\right]$ be an integer and write

$$
\begin{aligned}
\mathrm{P}(T \in & {\left.\left[j x u^{1-\varepsilon},(j+1) x u^{1-\varepsilon}\right]\right) } \\
= & \mathrm{P}\left(T \in\left[j x u^{1-\varepsilon},(j+1) x u^{1-\varepsilon}\right], S_{\left\lfloor(j+1) x u^{1-\varepsilon}\right\rfloor}-S_{T}>-u^{1-\varepsilon^{\prime}}\right) \\
& \quad+\mathrm{P}\left(T \in\left[j x u^{1-\varepsilon},(j+1) x u^{1-\varepsilon}\right], S_{\left\lfloor(j+1) x u^{1-\varepsilon}\right\rfloor}-S_{T} \leq-u^{1-\varepsilon^{\prime}}\right) .
\end{aligned}
$$

Denote by $P_{1}(u, j)$ the first probability and by $P_{2}(u, j)$ the second probability on the right-hand side of (4.5). For $P_{1}(u, j)$, we have

$$
\begin{aligned}
P_{1}(u, j) & \leq \mathrm{P}\left(S_{\left\lfloor(j+1) x u^{1-\varepsilon}\right\rfloor}>u-u^{1-\varepsilon^{\prime}}\right) \\
& \leq \mathrm{P}\left(S_{\left\lfloor(j+1) x u^{1-\varepsilon}\right\rfloor}>\left\lfloor(j+1) x u^{1-\varepsilon}\right\rfloor(1-\varepsilon) / x\right) \\
& \leq u^{-(1-2 \varepsilon) \bar{R}_{S}((1-\varepsilon) / x)}
\end{aligned}
$$

for large $u$. The upper bound is uniform for $j \in\left[0, u^{\varepsilon}-1\right]$. To estimate $P_{2}(u, j)$, observe that

$$
\begin{gathered}
\left\{T \in\left[j x u^{1-\varepsilon},(j+1) x u^{1-\varepsilon}\right], S_{\left\lfloor(j+1) x u^{1-\varepsilon}\right\rfloor}-S_{T} \leq-u^{1-\varepsilon^{\prime}}\right\} \\
\subseteq\left\{\sum_{i=\left\lfloor j x u^{1-\varepsilon}\right\rfloor+1}^{\left\lfloor(j+1) x u^{1-\varepsilon}\right\rfloor}\left|X_{i}\right| \mathbf{1}\left(X_{i} \leq 0\right) \geq u^{1-\varepsilon^{\prime}}\right\} .
\end{gathered}
$$


For $t>0$, we conclude as in (4.3) and (4.4) that, for large $u$,

$$
P_{2}(u, j) \leq u^{1-t\left(\varepsilon-\varepsilon^{\prime}\right)}
$$

for every $j$. If we choose $t$ to be large and combine (4.7) with (4.5) and (4.6), we see that

$$
\mathrm{P}\left(T \in\left[j x u^{1-\varepsilon},(j+1) x u^{1-\varepsilon}\right]\right) \leq u^{-(1-3 \varepsilon) \bar{R}_{S}((1-\varepsilon) / x)}
$$

for every $j \in\left[0, u^{\varepsilon}-1\right]$. By summing over $j$, we conclude that (2.6) holds. This completes the proof of Theorem 2.1.

Before proving Theorem 2.2, we state two technical lemmas. Let the family $\left\{\xi_{v}, v \in \mathbb{R}\right\}$, the process $\left\{S_{n}\right\}$, and the parameter $p(a)$ be as described in Section 2.2.

Lemma 4.1. Assume that (2.8) is satisfied for some $t>1$. For $v \in \mathbb{R}, n \in \mathbb{N}$, and $\delta>0$, let

$$
\underline{\xi}_{v}=\underline{\xi}_{v}(\delta, n)=\xi_{v} \mathbf{1}\left(\xi_{v} \leq n^{1-\delta}\right)
$$

and

$$
h=h(\delta, n)=n^{-1+\delta / 2} .
$$

Then, for a small $\delta$ and a given $\varepsilon>0$, there exists an $\varepsilon^{\prime} \in(0, \varepsilon)$ such that, for large $n$,

$$
\mathrm{E}\left(\mathrm{e}^{h \underline{\xi}_{v}}\right) \leq \mathrm{e}^{h\left(\left(1+\varepsilon^{\prime}\right) \mu(v)+\varepsilon\right)}
$$

for every $v \in \mathbb{R}$.

Lemma 4.2. Assume that (2.8) is satisfied for some $t>1$. Then, for every $a^{\prime}>a$ and $\delta>0$,

$$
\limsup _{n \rightarrow \infty}(\log n)^{-1} \log \mathrm{P}\left(S_{n}>n a^{\prime}, M_{n} \leq n^{1-\delta}\right) \leq-p(a) .
$$

Proof of Lemma 4.1. We begin by showing that, for a given $\varepsilon^{\prime \prime}>0$, we can choose $b>1$ such that

$$
\mathrm{E}\left(\max \left(\xi_{v},-b\right)\right)<\mu(v)+\varepsilon^{\prime \prime}
$$

for every $v \in \mathbb{R}$. For $b>1$, we have

$$
\mathrm{E}\left(\max \left(\xi_{v},-b\right)\right)=\mu(v)+\int_{b}^{\infty} \mathrm{P}\left(\xi_{v}<-x\right) \mathrm{d} x .
$$

Let $t>1$ be such that (2.8) holds. For every $x>0$, we then have

$$
\mathrm{E}\left(\left|\xi_{v}\right|^{t} \mathbf{1}\left(\xi_{v} \leq 0\right)\right) \geq x^{t} \mathrm{P}\left(\xi_{v}<-x\right)
$$

Thus, the integral in (4.11) tends to 0 when $b$ tends to infinity. This proves (4.10).

Let $b>1$ be such that (4.10) holds for every $v \in \mathbb{R}$. Write

$$
\underline{\xi}_{v}^{\prime}=\max \left(\underline{\xi}_{v},-b\right)+b .
$$

Observe that $\underline{\xi}_{v}^{\prime}$ is nonnegative and that $\underline{\xi}_{v}^{\prime} \leq n^{1-\delta}+b$. Let $\underline{\mu}^{\prime}(v)=\mathrm{E}\left(\underline{\xi}_{v}^{\prime}\right)$. Following Fuc and Nagaev (1971), write

$$
\mathrm{E}\left(\mathrm{e}^{h \underline{\xi}_{v}^{\prime}}\right)=\mathrm{E}\left(\frac{\mathrm{e}^{h \underline{\xi}_{v}^{\prime}}-1}{\underline{\xi}_{v}^{\prime}} \underline{\xi}_{v}^{\prime} \mathbf{1}\left(\underline{\xi}_{v}^{\prime}>0\right)\right)+1 .
$$


From its Taylor expansion, it is seen that $\left(\mathrm{e}^{h x}-1\right) / x$ is increasing in $x$ for $x \in(0, \infty)$. Thus,

$$
\mathrm{E}\left(\mathrm{e}^{h \underline{\xi}_{v}^{\prime}}\right) \leq \mathrm{E}\left(\frac{\mathrm{e}^{h\left(n^{1-\delta}+b\right)}-1}{n^{1-\delta}+b} \underline{\xi}_{v}^{\prime}\right)+1=\frac{\mathrm{e}^{h\left(n^{1-\delta}+b\right)}-1}{n^{1-\delta}+b} \underline{\mu}^{\prime}(v)+1 .
$$

Now, $h\left(n^{1-\delta}+b\right)$ tends to 0 as $n$ tends to infinity. Hence,

$$
\mathrm{E}\left(\mathrm{e}^{h \underline{\xi}_{v}^{\prime}}\right) \leq\left(1+\frac{\varepsilon^{\prime \prime}}{b}\right) h \underline{\mu^{\prime}}(v)+1 \leq \exp \left(\left(1+\frac{\varepsilon^{\prime \prime}}{b}\right) h \underline{\mu^{\prime}}(v)\right)
$$

for large $n$. By (4.10) and (4.12), we have

$$
\mathrm{E}\left(\mathrm{e}^{h \underline{\xi}_{v}}\right) \leq \mathrm{E}\left(\mathrm{e}^{h \underline{\xi}_{v}^{\prime}}\right) \mathrm{e}^{-h b} \leq \exp \left(h\left(\left(1+\frac{\varepsilon^{\prime \prime}}{b}\right)\left(\mu(v)+\varepsilon^{\prime \prime}+b\right)-b\right)\right),
$$

and an appropriate choice of $\varepsilon^{\prime \prime}$ shows that (4.8) is satisfied.

Proof of Lemma 4.2. Let $\underline{\xi}_{v}$ and $h$ be as in Lemma 4.1, and let $\delta, \varepsilon$, and $\varepsilon^{\prime}$ be positive real numbers such that (4.8) holds for large $n$ for every $v$. Write

$$
\underline{X}_{n}=X_{n} \mathbf{1}\left(X_{n} \leq n^{1-\delta}\right)
$$

and $\underline{S}_{n}=\underline{X}_{1}+\cdots+\underline{X}_{n}$. Furthermore, for $a \in \mathbb{R}$, write

$$
A_{n}(a)=\left\{\left(v_{1}, \ldots, v_{n}\right) \in \mathbb{R}^{n}: \mu\left(v_{1}\right)+\cdots+\mu\left(v_{n}\right) \leq n a\right\}
$$

and let $a^{\prime}>a$ be fixed. By Chebyshev's inequality, we have

$$
\mathrm{P}\left(\underline{S}_{n}>n a^{\prime}, \mu\left(\eta_{1}\right)+\cdots+\mu\left(\eta_{n}\right) \leq n a\right) \leq \mathrm{e}^{-h n a^{\prime}} \mathrm{E}\left(\mathrm{e}^{h \underline{S}_{n}} \mathbf{1}\left(\mu\left(\eta_{1}\right)+\cdots+\mu\left(\eta_{n}\right) \leq n a\right)\right)
$$

and, by (2.7), we have

$$
\begin{aligned}
& \mathrm{E}\left(\mathrm{e}^{h \underline{S}_{n}}\right.\left.\mathbf{1}\left(\mu\left(\eta_{1}\right)+\cdots+\mu\left(\eta_{n}\right) \leq n a\right)\right) \\
& \quad=\int_{\left(v_{1}, \ldots, v_{n}\right) \in A_{n}(a)} \mathrm{E}\left(\mathrm{e}^{h \underline{\xi}_{1}}\right) \cdots \mathrm{E}\left(\mathrm{e}^{h \underline{\xi}_{v_{n}}}\right) \mathrm{d} H_{n}\left(v_{1}, \ldots, v_{n}\right) .
\end{aligned}
$$

By the choice of $\delta, \varepsilon$, and $\varepsilon^{\prime}$, the integrand in (4.14) is bounded from above by

$$
\exp \left(h n \varepsilon+h\left(1+\varepsilon^{\prime}\right) \sum_{i \leq n} \mu\left(v_{i}\right)\right) \leq \exp \left(h n\left(\varepsilon+\left(1+\varepsilon^{\prime}\right) a\right)\right)
$$

on $A_{n}(a)$. By (4.13),

$$
\mathrm{P}\left(\underline{S}_{n}>n a^{\prime}, \mu\left(\eta_{1}\right)+\cdots+\mu\left(\eta_{n}\right) \leq n a\right) \leq \exp \left(h n\left(\varepsilon+\left(1+\varepsilon^{\prime}\right) a-a^{\prime}\right)\right) .
$$

For small $\varepsilon$ and $\varepsilon^{\prime}$, the exponent in (4.15) is negative, so, because $h n=n^{\delta / 2}$, we conclude that

$$
\limsup _{n \rightarrow \infty}(\log n)^{-1} \log \mathrm{P}\left(\underline{S}_{n}>n a^{\prime}, \mu\left(\eta_{1}\right)+\cdots+\mu\left(\eta_{n}\right) \leq n a\right)=-\infty .
$$

It follows that

$$
\limsup _{n \rightarrow \infty}(\log n)^{-1} \log \mathrm{P}\left(\underline{S}_{n}>n a^{\prime}\right) \leq-p(a),
$$

which proves (4.9) because

$$
\mathrm{P}\left(S_{n}>n a^{\prime}, M_{n} \leq n^{1-\delta}\right) \leq \mathrm{P}\left(\underline{S}_{n}>n a^{\prime}\right) .
$$


Proof of Theorem 2.2. For $a>0$ and $a^{\prime}>a$, we have

$$
\mathrm{P}\left(S_{n}>n a^{\prime}\right) \leq \mathrm{P}\left(S_{n}>n a^{\prime}, M_{n} \leq n^{1-\delta}\right)+\mathrm{P}\left(M_{n}>n^{1-\delta}\right) .
$$

It follows, from (4.1) and Lemma 4.2, that

$$
\limsup _{n \rightarrow \infty}(\log n)^{-1} \log \mathrm{P}\left(S_{n}>n a^{\prime}\right) \leq-\min \left(p(a), \bar{R}_{M}\right) .
$$

Thus, $\bar{R}_{S}(y) \geq \min \left(p(a), \bar{R}_{M}\right)$ for every $y>a^{\prime}$ and, hence, for every $y>a$. Condition (2.5) is satisfied by (2.8), meaning that, by Theorem 2.1, (2.9) holds for every $x<1 / a$.

Proof of Theorem 2.3. As in the proof of (2.6), the only case we consider in detail is the one in which $\bar{R}_{M} \in(0, \infty)$. By choosing $a \in(0,1)$ and applying Theorem 2.2, we see that (2.9) holds with $x=1$ and $\bar{R}_{M} \leq p(a)$. Thus, by (4.1), we must show that

$$
\limsup _{u \rightarrow \infty}(\log u)^{-1} \log \mathrm{P}(T \in(u, \infty)) \leq-\bar{R}_{M} .
$$

Fix a small $\varepsilon>0$ and a small $\delta>0$. By choosing $a^{\prime}=0$ in Lemma 4.2 and using (2.11), we see that

$$
\limsup _{n \rightarrow \infty}(\log n)^{-1} \log \mathrm{P}\left(S_{n}>0, M_{n} \leq n^{1-\delta}\right) \leq-\bar{R}_{M}-1 .
$$

Let $y \geq 1$. We start with the inequality

$$
\begin{aligned}
\mathrm{P}\left(T \in\left[u^{y}, u^{y+\delta}\right]\right) \leq & \mathrm{P}\left(S_{n}>0 \text { for some } n \in\left[u^{y}, u^{y+\delta}\right], M_{\left\lfloor u^{y+\delta}\right\rfloor} \leq u^{y(1-\delta)}\right) \\
& +\mathrm{P}\left(S_{n}>0 \text { for some } n \in\left[u^{y}, u^{y+\delta}\right], M_{\left\lfloor u^{y+\delta}\right\rfloor}>u^{y(1-\delta)}\right) .
\end{aligned}
$$

Denote by $Q_{1}(u, y)$ the first probability and by $Q_{2}(u, y)$ the second probability on the righthand side of (4.18). Let $u$ be large. Then, by (4.17), we have

$$
Q_{1}(u, y) \leq \sum_{n \in\left[u^{y}, u^{y+\delta}\right]} \mathrm{P}\left(S_{n}>0, M_{n} \leq n^{1-\delta}\right) \leq u^{y+\delta} u^{-y\left(\bar{R}_{M}+1-\epsilon\right)}=u^{\delta-y\left(\bar{R}_{M}-\epsilon\right)} .
$$

The estimate is uniform for $y \geq 1$. For $Q_{2}(u, y)$, we have

$$
Q_{2}(u, y) \leq \mathrm{P}\left(M_{\left\lfloor u^{y+\delta}\right\rfloor}>u^{y(1-\delta)}\right) \leq \mathrm{P}\left(M_{\left\lfloor u^{y+\delta}\right\rfloor}>\left\lfloor u^{y+\delta}\right\rfloor^{\frac{y(1-\delta)}{y+\delta}}\right) \leq u^{-y\left(\bar{R}_{M}-\varepsilon\right)}
$$

for small $\delta>0$. This estimate also holds uniformly for $y \geq 1$. By (4.18), (4.19), and (4.20),

$$
\begin{aligned}
\mathrm{P}(T \in(u, \infty)) & \leq \sum_{j=0}^{\infty} \mathrm{P}\left(T \in\left[u^{1+j \delta}, u^{1+(j+1) \delta}\right]\right) \\
& \leq u^{-\bar{R}_{M}+\delta+\varepsilon} \sum_{j=0}^{\infty} u^{-j \delta\left(\bar{R}_{M}-\varepsilon\right)}+u^{-\bar{R}_{M}+\varepsilon} \sum_{j=0}^{\infty} u^{-j \delta\left(\bar{R}_{M}-\varepsilon\right)} .
\end{aligned}
$$

For small $\varepsilon$, both of the above series are convergent, meaning that (4.16) follows from (4.1).

Proof of Theorem 2.4. Let $t>1$ be such that (2.14) holds. For $x \geq 0$, we have $1-x \leq \mathrm{e}^{-x}$. Thus, for every $y>0$ and $n \in \mathbb{N}$,

$$
\mathrm{P}\left(M_{n}>n^{y}\right) \geq \int_{v_{1}, \ldots, v_{n} \in \mathbb{R}}\left(1-\exp \left(-\sum_{i \leq n} \mathrm{P}\left(\xi_{v_{i}}>n^{y}\right)\right)\right) \mathrm{d} H_{n}\left(v_{1}, \ldots, v_{n}\right) .
$$


By Chebyshev's inequality,

$$
\sum_{i \leq n} \mathrm{P}\left(\xi_{v_{i}}>n^{y}\right) \leq D(t) n^{1-t y},
$$

where $D(t)$ is the supremum in (2.14). Thus, for $y$ close to 1 , we have

$$
\begin{aligned}
\mathrm{P}\left(M_{n}>n^{y}\right) & \geq(1+o(1)) \int_{v_{1}, \ldots, v_{n} \in \mathbb{R}} \sum_{i \leq n} \mathrm{P}\left(\xi_{v_{i}}>n^{y}\right) \mathrm{d} H_{n}\left(v_{1}, \ldots, v_{n}\right) \\
& =(1+o(1)) \sum_{i \leq n} \mathrm{P}\left(X_{i}>n^{y}\right)
\end{aligned}
$$

where $o(1)$ tends to 0 as $n$ tends to infinity. This and (2.13) imply (2.15).

\section{Technical background for the examples}

\subsection{Background for Example 3.1}

Let $F_{n}$ be the distribution function of $X_{n}$, for $n \in \mathbb{N}$. In the framework of Section 2.2, take $\mathrm{P}\left(\eta_{n}=n\right)=1$ and $G_{n}=F_{n}$. Then $\mu\left(\eta_{n}\right)=m_{n}$ for every $n$. Clearly, $p(a)=\infty$ for $a>m$. The required upper bounds follow from Theorems 2.2 and 2.3, and the lower bounds follow from Theorem 2.1. Now, assume that $X_{1}, X_{2}, \ldots$ are identically distributed, with distribution function $F$. We have

$$
\bar{\alpha}_{F}=\sup \left\{t \geq 0: \mathrm{E}\left(X_{1}^{t} \mathbf{1}\left(X_{1} \geq 0\right)\right)<\infty\right\}
$$

see, for example, Rolski et al. (1999, p. 39). We apply Theorem 2.4 to conclude that $\bar{R}_{M}=$ $\bar{\alpha}_{F}-1$ and $\underline{R}_{M}=\underline{\alpha}_{F}-1$; see also Hu and Nyrhinen (2004, Lemma 3.1). The limit on the left-hand side of (2.3) equals $1-\alpha_{-}$, where

$$
\alpha_{-}=\sup \left\{t \geq 0: \mathrm{E}\left(\left|X_{1}\right|^{t} \mathbf{1}\left(X_{1} \leq 0\right)\right)<\infty\right\}
$$

see Hu and Nyrhinen (2004, Theorems 2.1 and 2.2). By (3.2), for $t>\underline{\alpha}_{F}$, condition (2.3) is satisfied. This proves (2.4) and (2.10). Upper bounds (3.1) and (2.12) are obtained by truncating the $X$-variables from below and by applying Theorems 2.2 and 2.3 , as above.

Now assume that $\bar{F}$ is regularly varying with index $\alpha$. Then $\bar{\alpha}_{F}=\underline{\alpha}_{F}=\alpha$; see, for example, Feller (1971, Section VIII.8). To prove (3.3), define the process $\left\{S_{n}^{\prime}\right\}$ according to

$$
S_{n}^{\prime}=\sum_{i \leq n^{x}} X_{i} .
$$

Let $T^{\prime}=T^{\prime}(u)$ be the time of ruin of the process $\left\{S_{n}^{\prime}\right\}$. Then

$$
\left\{T \leq u^{x}\right\}=\left\{T^{\prime} \leq u\right\} .
$$

Let $S_{0}^{\prime}=0$ and $X_{n}^{\prime}=S_{n}^{\prime}-S_{n-1}^{\prime}$ for $n \in \mathbb{N}$. Take $\eta_{n}=0$ if $X_{n}^{\prime} \equiv 0$ and $\eta_{n}=n$ otherwise. Let $G_{n}=F$ for $n \geq 1$ and $G_{0}(x)=\mathbf{1}(x \geq 0)$ for $x \in \mathbb{R}$. Limit (3.3) then follows as above, from the application of Theorems 2.1 and 2.2 to the process $\left\{S_{n}^{\prime}\right\}$. Associated with $\left\{S_{n}^{\prime}\right\}$, the parameter $m$ is at most 0 and the parameter $p(a)$ is infinite for every $a>0$. 


\subsection{Background for Example 3.2}

Observe that $\left\{\left(\eta_{n}, \mu\left(\eta_{1}\right)+\cdots+\mu\left(\eta_{n}\right)\right)\right\}$ is also a Markov additive process. Therefore, for every $a>m, \mathrm{P}\left(\mu\left(\eta_{1}\right)+\cdots+\mu\left(\eta_{n}\right)>n a\right)$ tends to 0 exponentially fast; see Dembo and Zeitouni (1998, Section 3.1.1). It follows that $p(a)=\infty$ for every $a>m$.

Consider the parameters $\bar{R}_{M}$ and $\underline{R}_{M}$. By making use of (5.1), we see that Theorem 2.4 can also be applied here. Let $y>0$. For every $j \in E, \mathrm{P}\left(\eta_{n}=j\right)$ tends to $\pi(j)$ as $n$ tends to infinity. Hence,

$$
\begin{aligned}
\sum_{i \leq n} \mathrm{P}\left(X_{i}>n^{y}\right) & =\sum_{i \leq n} \sum_{j \in E} \mathrm{P}\left(\eta_{i}=j\right) \mathrm{P}\left(X_{i}>n^{y} \mid \eta_{i}=j\right) \\
& =\sum_{j \in E} \bar{G}_{j}\left(n^{y}\right) \sum_{i \leq n} \mathrm{P}\left(\eta_{i}=j\right) \\
& \sim n \sum_{j \in E} \pi(j) \bar{G}_{j}\left(n^{y}\right)
\end{aligned}
$$

as $n$ tends to infinity. By irreducibility, we have $\pi(j)>0$ for each $j$. Thus, $\bar{R}_{M}$ and $\underline{R}_{M}$ are as stated in Section 3. The required results follow from Theorems 2.1, 2.2, and 2.3.

\subsection{Background for Example 3.3}

Let $a>\mu(2)$. Then $\mathrm{P}\left(\mu\left(\eta_{1}\right)+\cdots+\mu\left(\eta_{n}\right)>n a\right)=0$ and, so, $p(a)=\infty$. By (5.1) and Theorem 2.4, we have $\bar{R}_{M}=\underline{R}_{M}=\alpha-1$. By Theorems 2.1 and 2.2, (1.2) holds with $R(x)=\alpha-1$ for every $x<1 / \mu(2)$.

Let $x>1 / \mu(2)$. Take $y<\mu(2)$ and fix a small $\varepsilon>0$ and $\delta_{1}, \delta_{2}$ such that $0<\delta_{1}<\delta_{2}<\varepsilon$. Let $\gamma=\mathrm{E}\left(v_{1}\right)$ and let $\left\{S_{n}^{\prime}\right\}$ be a random walk with the increment distribution $G_{2}$. Clearly,

$$
\begin{aligned}
\mathrm{P}\left(S_{n}>n y\right) \geq & \sum_{i \in\left[\delta_{1} n, \delta_{2} n\right]} \mathrm{P}\left(v_{1}+\cdots+v_{2 i} \leq n \varepsilon, v_{2 i+1}>n, S_{n}>n y\right) \\
\geq & \sum_{i \in\left[\delta_{1} n, \delta_{2} n\right]} \mathrm{P}\left(v_{1}+\cdots+v_{2 i} \leq n \varepsilon, S_{v_{1}+\cdots+v_{2 i}}>n \gamma \delta_{2}(\mu(1)+\mu(2)-\varepsilon)\right) \\
& \times \mathrm{P}\left(v_{2 i+1}>n\right) \min _{j \leq n \varepsilon} \mathrm{P}\left(S_{n}^{\prime}-S_{j}^{\prime}>n\left(y-\gamma \delta_{2}(\mu(1)+\mu(2)-\varepsilon)\right)\right) .
\end{aligned}
$$

By the law of large numbers, the magnitude of the last sum is $\left(\delta_{2}-\delta_{1}\right) n \mathrm{P}\left(v_{1}>n\right)$ for large $n$ and small $\varepsilon$ and $\delta_{2}$. It follows that

$$
\liminf _{n \rightarrow \infty}(\log n)^{-1} \log \mathrm{P}\left(S_{n}>n y\right) \geq 1-\beta
$$

for every $y<\mu(2)$. By (2.4), we have

$$
\liminf _{u \rightarrow \infty}(\log u)^{-1} \log \mathrm{P}(T \leq x u) \geq 1-\beta
$$

for every $x>1 / \mu(2)$.

Consider the reverse of inequality (5.2). We apply Theorem 2.2. As observed above, $\bar{R}_{M}=\alpha-1$. Consider $p(a)$ for $a \geq 0$, and let

$$
N=N(n)=\inf \left\{i: v_{1}+\cdots+v_{i} \geq n\right\}
$$


for $n \in \mathbb{N}$. By our assumptions, we can fix an $\varepsilon>0$ such that $\mu\left(\eta_{1}\right)+\cdots+\mu\left(\eta_{n}\right) \leq 0$ whenever the number of good years up to time $n$ is at least $n\left(\frac{1}{2}-\varepsilon\right)$. This is the case in the event

$$
\left\{\sum_{i \leq N / 2+1} v_{2 i-1} \leq n\left(\frac{1}{2}+\varepsilon\right)\right\} .
$$

Hence, for a given $\varepsilon^{\prime}>0$, we have

$$
\begin{aligned}
\mathrm{P}\left(\mu\left(\eta_{1}\right)+\cdots+\mu\left(\eta_{n}\right)>0\right) & \leq \mathrm{P}\left(\sum_{i \leq N / 2+1} \nu_{2 i-1}>n\left(\frac{1}{2}+\varepsilon\right)\right) \\
& \leq \mathrm{P}\left(\sum_{i \leq n\left(\gamma^{-1}+\varepsilon^{\prime}\right) / 2+1} v_{2 i-1}>n\left(\frac{1}{2}+\varepsilon\right)\right)+\mathrm{P}\left(N \geq n\left(\gamma^{-1}+\varepsilon^{\prime}\right)\right) .
\end{aligned}
$$

For a small $\varepsilon^{\prime}$, we apply Example 3.1 with $m>0$ to the first probability on the right-hand side of (5.3). The second such probability tends to 0 exponentially fast. This is seen by truncating the $\nu$-variables from above and applying Nyrhinen (1995, Theorem 4). It follows that $p(0) \geq \beta-1$. Hence, $p(a) \geq \beta-1$ for every $a \geq 0$. Recall that $\beta<\alpha$. By Theorem 2.2, we have

$$
\limsup _{u \rightarrow \infty}(\log u)^{-1} \log \mathrm{P}(T \leq x u) \leq 1-\beta .
$$

This and (5.2) imply (1.2) with $R(x)=\beta-1$ for every $x>1 / \mu(2)$.

\subsection{Background for Example 3.4}

By (3.4) and Nyrhinen (2001, Theorem 2), (3.5) holds for every $x>1 / c^{\prime}(\alpha)$. It is easy to see that $\rho$ is continuous, meaning that it suffices to prove (3.5) for every $x<1 / c^{\prime}(\alpha)$.

Let $x<1 / c^{\prime}(\alpha)$ be fixed. Choose a large $n_{0} \in \mathbb{N}$ and define the process $\left\{S_{n}^{\prime}\right\}$ by

$$
S_{n}^{\prime}= \begin{cases}X_{1}+\cdots+X_{n} & \text { for } n \leq n_{0}, \\ S_{n_{0}}+\sum_{i \in\left[n_{0}+1, x \log n\right]} X_{i} & \text { for } n>n_{0} .\end{cases}
$$

Choosing a large $n_{0}$ guarantees that every increment of $\left\{S_{n}^{\prime}\right\}$ is either identically 0 or equals $X_{i}$ for some $i$. Let $T^{\prime}=T^{\prime}(u)$ be the time of ruin of the process $\left\{S_{n}^{\prime}\right\}$. For large $u$, we have

$$
\{T \leq x \log u\}=\left\{T^{\prime} \leq u\right\} .
$$

Let $S_{0}^{\prime}=0$ and $X_{n}^{\prime}=S_{n}^{\prime}-S_{n-1}^{\prime}$ for $n \in \mathbb{N}$, and write

$$
M_{n}^{\prime}=\max \left(X_{1}^{\prime}, \ldots, X_{n}^{\prime}\right) .
$$

Let $\bar{R}_{M^{\prime}}(x)$ and $\underline{R}_{M^{\prime}}(x)$ be the parameters (2.1) and (2.2) associated with $\left\{M_{n}^{\prime}\right\}$. We will show that

$$
\bar{R}_{M^{\prime}}(x)=\underline{R}_{M^{\prime}}(x)=\rho(x) .
$$

Let $\delta>0$. For $z \in(0,1)$ and large $n$, we have

$$
\begin{aligned}
\mathrm{P}\left(M_{n}^{\prime}>n^{1+\delta}\right) & \geq \mathrm{P}\left(A_{1} \cdots A_{\lfloor x \log n\rfloor-1}>n^{z}, B_{\lfloor x \log n\rfloor}>n^{1-z+\delta}\right) \\
& =\mathrm{P}\left(\log A_{1}+\cdots+\log A_{\lfloor x \log n\rfloor-1}>z \log n\right) \mathrm{P}\left(B_{1}>n^{1-z+\delta}\right) .
\end{aligned}
$$


By Cramér's theorem,

$$
\liminf _{n \rightarrow \infty}(\log n)^{-1} \log \mathrm{P}\left(M_{n}^{\prime}>n^{1+\delta}\right) \geq-x c^{*}(z / x)-\alpha(1-z+\delta)
$$

see Dembo and Zeitouni (1998, Theorem 2.2.3). By choosing $z=x c^{\prime}(\alpha)$ and letting $\delta$ tend to 0 , we see that $\underline{R}_{M^{\prime}}(x) \leq \rho(x)$. To obtain the reverse inequality, write

$$
Y_{n}=\log \left(1+B_{n} \mathbf{1}\left(B_{n} \geq 0\right)\right)+\sum_{i \leq n-1} \log A_{i}
$$

for $n \in \mathbb{N}$. Then, for large $n$,

$$
\mathrm{P}\left(M_{n}^{\prime}>n^{1-\delta}\right) \leq \mathrm{P}\left(Y_{i}>(1-\delta) \log n \text { for some } i \leq x \log n\right) .
$$

This is a finite-time ruin probability associated with the process $\left\{Y_{n}\right\}$. Write

$$
c_{Y}(t)=\limsup _{n \rightarrow \infty} n^{-1} \log \mathrm{E}\left(\mathrm{e}^{t Y_{n}}\right)
$$

for $t \in \mathbb{R}$. By (5.1), we have $c_{Y}(t)=c(t)$ for $t \in(0, \alpha)$ and $c_{Y}(t)=\infty$ for $t>\alpha$. Furthermore,

$$
c_{Y}^{*}(v)=\alpha v-c(\alpha)
$$

for $v \geq c^{\prime}(\alpha)$. It follows from Nyrhinen (1998, Theorem 2.2) that, for $\delta$ close to 0 , we have

$$
\limsup _{n \rightarrow \infty}(\log n)^{-1} \log \mathrm{P}\left(M_{n}^{\prime}>n^{1-\delta}\right) \leq-\alpha(1-\delta)+x c(\alpha) .
$$

Thus, $\bar{R}_{M^{\prime}}(x) \geq \rho(x)$ and (5.4) holds.

For a given $\delta>0$ and large $u$, we have

$$
\{T \leq x \log u\} \subseteq\left\{(x \log u) M_{\lfloor u\rfloor+1}^{\prime}>u\right\} \subseteq\left\{M_{\lfloor u\rfloor+1}^{\prime}>u^{1-\delta}\right\} .
$$

It follows that

$$
\limsup _{u \rightarrow \infty}(\log u)^{-1} \log \mathrm{P}(T \leq x \log u) \leq-\rho(x) .
$$

For the reverse inequality, we apply Lemma 2.1 to $\left\{S_{n}^{\prime}\right\}$. Observe that

$$
\left\{\sum_{i \leq n}\left|X_{i}^{\prime}\right| \mathbf{1}\left(X_{i}^{\prime} \leq 0\right)>n^{1+\delta}\right\}=\left\{\sum_{i \leq x \log n}\left|B_{i}\right| \mathbf{1}\left(B_{i} \leq 0\right) A_{1} \cdots A_{i-1}>n^{1+\delta}\right\}
$$

for large $n$. Let

$$
\alpha_{-}=\sup \left\{t: \mathrm{E}\left(\left|B_{1}\right|^{t} \mathbf{1}\left(B_{1} \leq 0\right)\right)<\infty\right\}
$$

and write

$$
\rho_{-}(x)= \begin{cases}\alpha_{-}-x c\left(\alpha_{-}\right) & \text {if } x \leq \frac{1}{c^{\prime}\left(\alpha_{-}\right)}, \\ x c^{*}\left(\frac{1}{x}\right) & \text { if } x \in\left(\frac{1}{c^{\prime}\left(\alpha_{-}\right)}, \frac{1}{c^{\prime}(w)}\right), \\ w & \text { if } x \geq \frac{1}{c^{\prime}(w)} .\end{cases}
$$


If $\alpha_{-}=\infty$ then the first case of (5.6) does not occur. By the first part of the proof, we have

$$
\limsup _{n \rightarrow \infty}(\log n)^{-1} \log \mathrm{P}\left(\sum_{i \leq n}\left|X_{i}^{\prime}\right| \mathbf{1}\left(X_{i}^{\prime} \leq 0\right)>n\right) \leq-\rho_{-}(x) .
$$

We have assumed that $\alpha_{-}>\alpha$. By observing that $\rho(x)=x c_{Y}^{*}(1 / x)$ for every $x<1 / c^{\prime}(w)$, we can easily check that $\rho_{-}(x)>\rho(x)$ for every $x<1 / c^{\prime}(\alpha)$. Thus, (2.3) is satisfied and (2.4) holds. This and (5.5) prove (3.5).

\section{References}

ASMUSSEN, S. (1998). Subexponential asymptotics for stochastic processes: extremal behaviour, stationary distributions and first passage probabilities. Ann. Appl. Prob. 8, 354-374.

Asmussen, S. AND KlüPpelberg, C. (1996). Large deviations results for subexponential tails, with applications to insurance risk. Stoch. Process. Appl. 64, 103-125.

Asmussen, S., Fløe Henriksen, L. AND KlüPPelberg, C. (1994). Large claims approximations for risk processes in a Markovian environment. Stoch. Process. Appl. 54, 29-43.

Asmussen, S., Schmidli, H. ANd SchmidT, V. (1999). Tail probabilities for non-standard risk processes with subexponential jumps. Adv. Appl. Prob. 31, 422-447.

CAI, J. AND TANG, Q. (2004). On max-sum equivalence and convolution closure of heavy-tailed distributions and their applications. J. Appl. Prob. 41, 117-130.

Collamore, J. F. (1998). First passage times of general sequences of random vectors: a large deviations approach. Stoch. Process. Appl. 78, 97-130.

Daykin, C. D., Pentikäinen, T. and Pesonen, M. (1994). Practical Risk Theory for Actuaries (Monogr. Statist. Appl. Prob. 53). Chapman and Hall, London.

Dembo, A. And Zeitouni, O. (1998). Large Deviations Techniques and Applications, 2nd edn. Springer, New York.

Duffy, K., Lewis, J. T. And Sullivan, W. G. (2003). Logarithmic asymptotics for the supremum of a stochastic process. Ann. Appl. Prob. 13, 430-445.

Embrechts, P. AND Veraverbeke, N. (1982). Estimates for the probability of ruin with special emphasis on the possibility of large claims. Insurance Math. Econom. 1, 55-72.

Embrechts, P., KlüPPelberg, C. And Mikosch, T. (1997). Modelling Extremal Events. Springer, Berlin.

Feller, W. (1971). An Introduction to Probability Theory and Its Applications, Vol. 2, 2nd edn. John Wiley, New York

Fuc, D. H. ANd Nagaev, S. V. (1971). Probability inequalities for sums of independent random variables. Theory Prob. Appl. 16, 643-660.

Hu, Y. (2004). Large deviations for generalized compound Poisson risk models and its bankruptcy moments. Sci. China Ser. A 47, 311-319.

Hu, Y. And Nyrhinen, H. (2004). Large deviations viewpoints for heavy-tailed random walks. J. Theoret. Prob. 17, 761-768.

KlÜPPELberg, C. AND STADTMÜLleR, U. (1998). Ruin probabilities in the presence of heavy-tails and interest rates. Scand. Actuarial J. 1998, 49-58.

Mikosch, T. AND SAmorodnitsky, G. (2000). The supremum of a negative drift random walk with dependent heavytailed steps. Ann. Appl. Prob. 10, 1025-1064.

Nyrhinen, H. (1995). On the typical level crossing time and path. Stoch. Process. Appl. 58, 121-137.

Nyrhinen, H. (1998). Rough descriptions of ruin for a general class of surplus processes. Adv. Appl. Prob. 30, 10081026.

Nyrhinen, H. (1999). On the ruin probabilities in a general economic environment. Stoch. Process. Appl. 83, 319-330.

Nyrhinen, H. (2001). Finite and infinite time ruin probabilities in a stochastic economic environment. Stoch. Process. Appl. 92, 265-285.

Paulsen, J. (2002). On Cramér-like asymptotics for risk processes with stochastic return on investments. Ann. Appl. Prob. 12, 1247-1260.

Rolski, T., Schmidli, H., Schmidt, V. And Teugels, J. (1999). Stochastic Processes for Insurance and Finance. John Wiley, Chichester.

Tang, Q. And Tsitsiashvili, G. (2003). Precise estimates for the ruin probability in finite horizon in a discrete-time model with heavy-tailed insurance and financial risks. Stoch. Process. Appl. 108, 299-325.

TANG, Q. AND TsitsiashviLi, G. (2004). Finite- and infinite-time ruin probabilities in the presence of stochastic returns on investments. Adv. Appl. Prob. 36, 1278-1299. 